\title{
The Effectiveness of Education for Family Management of Asthma in Children: A Preliminary Report
}

\author{
Noreen M. Clark, Ph.D. \\ University of Michigan, School of Public Health \\ Charles H. Feldman, M.D. \\ David Evans, Ph.D. \\ E. Joel Millman, Ph.D. \\ Yvonne Wailewski, M.S. \\ Iraida Valle, M.S \\ Columbia University, College of Physicians and Surgeons
}

ABSTRACT: This paper reports preliminary findings in a convenience sample of one half the population enrolled in a study of self-management in low income families where a child has asthma. In initial evaluation data, parents participating in self-management education reported significantly less fear and anxiety associated with their children's wheezing episodes than did control families. They also reported that their children exhibited fewer signs of stress during a wheezing episode. A trend toward reduced school absences and emergency room visits was noted among participating families. The actions taken by a family to manage the illness increased with the number of sessions attended.

In a previous issue of the Quarterly (Vol. No. 4, Winter 1980), we described the study design and methods of our research on the management of pediatric asthma in low income families. We reported in detail, the development of self-management education for children with asthma and their parents. The education was implemented following an extensive needs assessment of health practices in the study families. Project evaluation entails collection of follow-up data at two points in time: at completion of the educational program and again six months later. We have begun the first phase of data collection, and in this paper we will present preliminary findings.

This study is supported by Contract No. 1-HR-7-2973 from the Lung Division, National Heart, Lung and Blood Institute. National Institutes of Health. Reprint requests should be addressed to the senior author, Noreen M. Clark at the University of Michigan. School of Public Health 1420 Washington Heights, Ann Arbor, Michigan 48109. 


\section{BACKGROUND}

The educational program was formulated on the basis of several hypotheses:

1. education would foster better family management of asthma;

2. improved family management would reduce the disruptive and stressful impact of asthma on the family;

3. better family management would decrease health care utilization, that is, hospitalizations and emergency room visits;

4. improved management would reduce school absences.

These deleterious effects of asthma have been previously noted. 1"

Several reports have confirmed the efficacy of education in the management of various chronic conditions."1" Investigators have recently focussed on educational programs in relation to asthma care.

Green and his colleagues found that a single group discussion session with adults having asthma subsequently reduced emergency room visits by $50 \%$ in comparison with a control group. ${ }^{13}$ The program achieved a cost-benefit ratio of 1:5. Weingarten and his associates replicated this one session educational intervention for children with asthma and their parents. ${ }^{14}$ Families in the experimental group who attended the single discussion session significantly reduced the frequency of emergency room visits during a nine-month follow-up period compared to controls.

Tobbins and Finkelstein also achieved a satisfactory outcome of reduced acute hospital admissions by developing a program for children with asthma and their parents. ${ }^{15}$ An asthma nurse specialist conducted bimonthly lectures for the parents on asthma management. Blumethal and his colleagues described a comprehensive family educational program but did not report significant findings based on objective outcome measures. ${ }^{16}$ Parents and children participated in educational sessions, and children were enrolled in physical education activities designed to increase physical capabilities and self-confidence.

Parcel and his colleagues devised a school-based health education program for children with asthma and evaluated it with a quasi-experimental design. ${ }^{17}$ This 24 session educational program significantly improved knowledge of asthma, increased the child's sense of control over this or her health, and reduced anxiety 
associated with illness. Creer and his colleagues designed a selfmanagement program for children with severe asthma using behavioral modification techniques to reduce the duration of hospitalization at the National Asthma Center. ${ }^{18}$ The findings reported have been limited to individual cases. These studies have shown that educational intervention is effective for those with asthma.

In the design of the research and demonstration program, we attempted to focus on considerations not addressed previously. No other program had included a study population of children with asthma from low income communities. Only two studies had used an experimental research design to evaluate outcome. In addition, most programs dealt with a limited number of management problems and a small sample population. Several investigators relied entirely on a single session intervention format. We aimed at a more comprehensive, multiple session program based on an initial assessement of learning needs from the perspective of the low income families. We employed an experimental research design to evaluate our results. We enrolled a sufficiently large study population (200 families by random assignment to experimental group and 100 to control group) to ensure statistical validity for follow-up evaluation data analysis. A review of pre-intervention data revealed no statistically significant differences between experimental and control groups for any outcome measurements.

As previously described, the educational program focused on six major management areas. Each core theme had been emphasized by the families during the needs assessment phase and served as the topic for a separate educational session in our six part series: (1) managing an asthma attack; (2) getting children to take medicine; (3) setting realistic limits on children's activities; (4) communicating with the physician; (5) managing asthma at school; (6) keeping a child healthy to prevent asthma. Parents and children had the opportunity to attend classes conducted in English and Spanish at the allergy clinics conducted in English and Spanish at the allergy clinics of four major metropolitan New York hospitals. The learning methodologies and activities employed are described in detail elsewhere. ${ }^{19}$

\section{PRELIMINARY FINDINGS}

More than one-half the total number of study families have been interviewed in the followup evaluation phase and we are able to report preliminary findings for 140 cases. 
It is very important to note that the 140 families represent a sample of convenience and only a portion of the total study population. Using these families maximizes the risk of selfselection. Because they were accessible, they were probably the most willing to be interviewed. They may well represent some characteristic in common that is different from the other segment of the population as yet not interviewed, that is, they may constitute a somewhat biased sample. Unless otherwise indicated, the data below represent comparisons between 97 study and 43 control group families and are reported by the primary caretaker (usually a parent) of the child with asthma.

Table 1 illustrates program effects on five dimensions of asthma management:

1. Experimental group parents reported taking more selfmanagement steps than control group parents in three situations: (a) treating mild wheezing ( 2.1 to $1.6 ; p<0.05)$; (b) controlling asthma attacks $(5.2$ to $4.6 ; p=0.08$ ); (c) routine preventive measures to avoid asthma symptoms (6.3 to 5.6; $p<0.01)$.

2. Experimental group parents described that their children suffered significantly fewer symptoms of emotional stress on a regular basis than did control group parents $(0.47$ to 1.03; $p<0.05$ ).

3. Experimental group parents were less fearful of their child's possible death during an asthma attack (25\% to $42 \%$; $\mathrm{p}<0.05$ ).

4. Fewer experimental group parents reported that their child missed a gym class because of asthma in the last year $(38 \%$ to $57 \%$ p $<0.05$ ).

5. The experimental group showed trends toward larger reductions than the control group from baseline to postintervention values in two parameters: (a) annual emergency room visits for asthma $(-4.4$ to $-3.9 ; \mathrm{p}=\mathrm{NS})$ (b) annual school absences $(-9.9$ to $-5.6 ; p=N S)$

Our pre-intervention data identified that as a group our study families engaged in a variety of relevant self-management practices. However, each family had a limited repertoire of skills. An emphasis of our program was to broaden this range of management practices. During the follow-up interview, parents were asked what they did to treat the symptoms of an asthma attack, a mild wheezing episode, and as a preventive measure on a regular basis to avoid an attack. As can be seen in Table 1 . experimental group parents engaged in more management 
1. Self-manarement activities

number of steps taken to treat mild wheezing

number of steps taken to treat asthma attacks

number of regular measures taken to prevent asthma symptoms

2. Reduced stress and disruptions

Percent of parents who fear their child might die during an attack

Number of symptoms of emotional stress experienced "often" by children as reported by their parents

Percent of parents reporting that their child had to miss gym class because of asthria

3. lealth care use and school absences

Annual emergency room visits: pre-intervention year intervention year difference scores
Experimental Control Significance* $N=97 \quad N=43$

$\begin{array}{lll}2.1 & 1.6 & \mathrm{p}<.05 \\ 5.2 & 4.6 & \mathrm{p}<.08 \\ 6.3 & 5.6 & \mathrm{p}<.01\end{array}$

$25 \%$

$42 \% \quad P=.05$

0.47

$1.03 \quad \mathrm{p} \cdot .05$

$38 \%$

$57 \% \quad$ P - . 05

$N=128 \quad N=50$

$\begin{array}{rr}7.7 & 7.2 \\ 3.7 & 3.7 \\ -4.4 & -3.9 \quad P=N S\end{array}$

\begin{abstract}
Annual school absences: pre-intervention year intervention year difference scores
\end{abstract}

Annual hospltalizations: pre-intervention year intervention year difference scores
28.2
18.7
-9.9

0.40

0.33

0.12
22.0

16.7

$-5.6 \quad \mathrm{P}=\mathrm{NS}$

0.47

0.49

$0,20 \quad \mathrm{P}=\mathrm{NS}$

*The t-test was used to test differences between mean values and the $x^{2}$ test was used to exanine differences in proportions.

practices than did the control group parents. The experimental group was significantly more likely to give fluids, use relaxation exercises, perform postural drainage and set limits on the child's activities for preventive purposes.

Caretakers were also asked about their own feelings during the child's attack and symptoms of emotional stress (nailbiting, stomach aches) experienced by their child with asthma. Experimental group parents reported a lower degree of fear and fewer syptoms of stress in their children. They also indicated that their children were less likely to be prevented by asthma from taking part in physical education activities at school

A preliminary comparison of parent's pre- to post-intervention reports of emergency room visits and school absences was 
conducted. Data from the first 178 post interviews were hand tabulated and compared with the pre-intervention reports of these parents. Both the 128 experimental group and 50 control group parents reported sharp reductions. The experimental group significantly decreased emergency room visits from 7.7 to 3.7 $(p<0.01)$, while the control group visits decreased from 7.2 to 3.7 $(p=N S)$. However, a t-test comparing the reductions of the experimental and control groups showed that the experimental group did not have a significantly greater decrease in emergency room visits than the control group.

Reductions in school absences followed the same pattern. The experimental families reported a significant decrease in absences from 28.2 to $18.7(p<0.01)$, while control families reduced absences from 22.0 to 16.7 ( =NS). However, a t-test comparing the experimental and control groups indicated that the reductions did not differ significantly.

The rate of hospitalization for asthma did not vary significantly from the baseline year to the intervention year for either the experimental or control group

In the follow-up interviews completed to date, we found no differences in parents' reports of problems related to getting their children to take asthma medicine or of the frequency or severity of wheezing episodes.

An important dimension of our study was to test the presumed link between attendance at more educational sessions and increased ability to self-manage. While $82 \%$ of the experimental group families attended one or more learning sessions $50 \%$ attended half or more. An analysis of covariance controlling for the number of educational sessions attended indicated that the self-management program specifically accounted for the differences found between the experimental and control groups Similarly, significant correlations between outcome measures and attendance suggest that multiple session programs may be capable of achieving more changes in asthma management practices than single session interventions. The ANCOCA controlling for the number of classes attended examined the relationship between attendance and program outcome measures. Significant correlations were obtained between the number of classes attended by adults and actions taken to control an attack $(r=.26$; $p<.01)$ and actions taken for mild wheezing $(r=.16 ; p<.05)$.

\section{DISCUSSION}

It is evident in these preliminary findings that the educational program has enhanced the families existing repertoire of asthma 
management skills. Not only have participants increased the number of self-management practices; they have incorporated practices of a sophisticated nature, such as postural drainage, and breathing exercises. One educational session specifically encouraged parents and children to negotiate more realistic limits on the child's activities. This session stressed removing asthma from the arena of family conflict by suggesting a variety of ways to promote normal activity while protecting the child from potential wheezing. That experimental group children were less likely to miss gym class despite the absence of any change in severity or frequency of wheezing suggests that their parents did encourage the child to take part in normal activities to a greater extent than control parents.

Our post-intervention data for both emergency room visits and school absences failed to show a statistically significant difference when we compared reductions between experimental and control groups, but the experimental group showed a trend toward greater reductions especially in school absences. At this point in our investigation we believe the lack of statistical significance to be error variability. It is likely our convenience sample includes a greater proportion of those families who more readily seek service at the health care facilities, the high users. The scores of these high users are likely to be more influential in our present data than they will be in the final results of the total study population. Their scores would tend to inflate estimates of the true variance and the true means when we compare scores of experimental and control groups.

We found no significant changes in hospitalizations in either group pre- to post-program. Although we predicted a decline, we consider hospitalizations to be a much less sensitive measure of the outcome of asthma self-management. It is likely that many episodes of severe wheezing cannot be managed at home and will frequently result in hospitalization.

As mentioned previously, there is little difference in the frequency and severity of wheezing reported by parents. Our program's impact seems to be on the feelings that families have about asthma and their ability to proceed with daily activities. It is likely that the emergency room has continued to be the alternative of families who were unsure and fearful about the acute episode.

The lack of difference in the frequency and severity of wheezing reported by families may be related to the fact that these low income families are subject to environmental conditions over which they have little or no control. As discussed in our earlier article, ${ }^{20}$ almost one quarter to one half of the families live in 
housing where the elevator is frequently out of order, the building condition is poor, apartments are beset with dampness and drafts; there is frequently no heat, and there are no air conditioners. These adverse conditions are likely to override the best of family management skills and precipitate frequent and of ten severe wheezing.

\section{CONCLUSION}

These post-intervention data have been derived from a preliminary phase of the program follow-up. On the basis of these gross measures, it appears that a comprehensive, multiple-session approach to asthma education has had significant impact on the management practices of families.

The preliminary results confirm the potential benefits of this non-medical treatment modality for children with asthma. It is possible to reach and educate low income families seeking care in the clinic setting. Families can learn and use sophisticated management techniques and significantly increase their repertoire of skills. The fear and stress associated with wheezing can be significantly reduced. It appears also that diminished stress and increased management ability may contribute to less frequent school absences. A link between higher levels of management and greater attendance at learning sessions has been established.

This evaluation research project has accumulated an extensive, diverse data set to which further observations will be added. We have not completed the analysis of all the outcome measures for the 140 post-intervention interviews reported above. When this analysis and other data collection procedures are complete, we will report the following additional data: (a) objective measures of health care utilization based on emergency room and in-patient hospital records; (b) objective measures of school absences based on each child's school records; (c) pulmonary function testing parameters from baseline and post-intervention periods; (d) data on physician's assessments on health status and asthma severity based on frequency, severity and duration of wheezing episodes, medication administration; (e) data based on parent and child interviews assessing asthma knowledge, general and asthmaspecific health beliefs, locus of control, measures of the child's adaptation to school, relationships with siblings and family, measures of the level of disruption and conflict in family life, degree of social support, and additional indices of selfmanagement capability. We believe, however, that the findings presented based on interviews with a convenience sample of the study population, illustrate two initial advantages of the asthma 
self-management program: (a) the ability to elicit active participation by the low income families who attend the allergy clinics; (b) the means to increase the range of self-management practices and achieve positive outcomes related to school absences and to the psychological status of child and parent.

\section{REFERENCES}

1. Pless I: Theoretical and practical considerations in the measurement of outcome In $\mathrm{G}$ Creuve \& I Pless (Eds). Chronic Childhood IIIness: Assessment of Outcome. Washıngton, D.C., United States Department of Health, Education, and Welfare, 1974

2. Davis D NIAID initiatives in allergy research. / Allergy Clin Immunol 49-323-328, 1971

3. National Institute of Allergy and Infectious Diseases Task Force: Asthma and other allergic diseases. NIH Publication No 70-397, 1979.

4. Pless I. Pinkerton P Chronic Childhood Disorder Promoting Patterns of Adjustment London, Henry Kempton Publishing, 1975

5. Creer T, Renne C Christian W: Behavioral contributions to rehabilitation and childhood asthma. Rehab Lit 37-226-232, 1976

6. Wright L: Councellıng with parents of chroncally ill children Postgraduate Medicine 47:173, 1970

7. Green L, Werlin S, Schauffler H: Research demonstration issues in self care Cambridge, Massachusetts, Arthur D. Little, Inc, 1976

8. Karetzky M: Asthma in the South Bronx: Clinical and epidemiological characteristics. I Allergy Clin Immunology 60:383-390, 1977

9. Miller L, Coldstein I More efficient care of diabetic patients in a county hospital setting. New England lournal of Medicine 286:1388-1391. 1972

10 Inul T Yourtee E Williamson I Improved outcomes in hypertension after physician tutorials Annals of Internal Medicine 84646-651, 1976

11. Rosenberg S: Patient education leads to better care for heart patients. HSMHA Rep 86:793-802, 1971

12. Levine P, Britten A: Supervised pattent management of hemophilia Annals of Internal Medicine 78:195-201, 1973

13. Green L, Werlin S, Schauffler $H$. Avery $C$ : Research and demonstration issues in selfcare: Measuring the decline of medicocentrism Health Education Monographs 5:161-189, 1977

14. Weingarten $\mathrm{V}$, Coodfriend $\mathrm{S}$, Harris $\mathrm{C}$. Health education demonstration project at Roosevelt Hospital Institute of Public Affarrs, New York, 1976

15. Robbins S, Finklestem J: Reducing the emotional and economic costs of hospitalization of acutely ill asthmatics Clin Pediatrics 12:550, 1973

16. Blumenthal M. Cushing R, Fasıngbauer $T$ : A community program for the management of bronchial asthma Annals of Allergy 30.391-398, 1972

17. Parcel $C$, Nadar $P$. Tiernan $K$ : A health education program for children with asthma. Journal of Developmental and Behavioral Pediatrics 1.128-132, 1980

18. Creer T, Burns K: Self-management trainıng for children with chronic bronchial asthma Psychother Psychosom 32:270-278, 1979

19. Clark N, Feldman C. Evans D. Millman 1. Wasilewskı Y, Valle I: A Program to Change Family Health Behavior: The Management of Pediatric Asthma (in preparation)

20. Clark N. Feldman C. Freudenberg N, Millman J. Wasilewski Y, Walle I: Developing Education for Children with Asthma through study of self-management behavior. Health Education Quarterly 7(4):278-297, Winter 1980 